\title{
Single Institutional Experience on Orbital Inflammatory Pseudotumor: Diagnostic and Management Challenge
}

\author{
Roshanak Derakhshandeh ${ }^{1}$, Yiannis Petros Dimopoulos ${ }^{1} \oplus$, Todd Alan Goodglick ${ }^{2} \oplus$, Joeffrey Chanine ${ }^{1} \oplus$, \\ Sina Sabet ${ }^{1}$, Metin Özdemirli ${ }^{1}$
}

${ }^{1}$ Department of Pathology and Laboratory Medicine, MedStar Georgetown University Hospital, USA

${ }^{2}$ Department of Ophthalmology, MedStar Georgetown University Hospital, USA

\begin{abstract}
Aims: Orbital inflammatory pseudotumor is considered a non-neoplastic inflammatory process. The finding of clonality of B or T-cell receptors in cases pathologically diagnosed as orbital inflammatory pseudotumor has unknown clinicopathologic significance. We sought to investigate potential B and T-cell clonality and concomitant diseases in cases pathologically diagnosed as orbital inflammatory pseudotumor. Methods: Cases diagnosed as orbital inflammatory pseudotumor at our institution were retrospectively analyzed. Hematoxylin and eosinstained slides, immunohistochemically stained slides and polymerase chain reactions on cell block material for the investigation of clonality of B and T-cell receptors were evaluated, to confirm the diagnosis and investigate the prevalence of concomitant diseases.

Results: A total of 13 cases showing characteristic histopathologic features of orbital inflammatory pseudotumor were identified. CD138, $\mathrm{IgG}$, and IgG4 showed varying numbers of plasma cells in each case,
\end{abstract}

with 5 cases $(5 / 13,38 \%)$ exhibiting relative increase in the presence of IgG4 plasma cells. However, no cases showed diagnostic findings of IgG4-related disease (IgG4-RD). polymerase chain reactions analysis showed clonal B-cell populations in 2 cases $(2 / 13,15 \%)$. No cases showed anaplastic lymphoma kinase expression by immunohistochemistry. There were no clinical reports of progression to lymphoma or development of systemic IgG4-RD in any of the patients (average follow-up of 300 days), with $38 \%$ of patients showing systemic autoimmune conditions.

Conclusion: A small but significant percentage of typical orbital inflammatory pseudotumor on histology showed B-cell clonality on polymerase chain reactions analysis of B-cell receptors, or features suggestive, but not diagnostic of IgG4-RD. Close follow-up of these patients to identify development of lymphoma, systemic IgG4-RD, or other rheumatologic conditions may be clinically warranted.

\section{INTRODUCTION}

Inflammatory pseudotumor (IP) is considered a benign idiopathic inflammatory process which most commonly involves the lung and the orbit, but has also been described in other sites of the body. IPs belong to a broad heterogenous group of disorders, ranging from benign inflammatory processes all the way to inflammatory myofibroblastic tumor (IMT), which is considered a neoplastic process showing clonal chromosomal abnormalities and leading to anaplastic lymphoma kinase (ALK) expression. ${ }^{1}$

The term IP was first described by Brunn in 1932; In 1954, Umiker and Iverson referred to this disease as "IP" due to its ability to mimic malignant tumors clinically and radiologically. ${ }^{2}$ Orbital inflammatory pseudotumor (OIP), also known as idiopathic orbital inflammatory syndrome, was first described in 1903 by Gleason, and characterized as a separate clinicopathological entity in 1905 by Birch-Hirschfeld. ${ }^{3}$

Orbital inflammatory pseudotumors represent an etiologically and pathogenetically heterogeneous group of diseases sharing similar pathologic features, including the formation of an orbital mass, chronic inflammation, vascular proliferation, and connective tissue hyperplasia, in the absence of specific etiologies. OIP may present as acute, subacute, or chronic. It is usually unilateral, but bilateral disease (simultaneous or sequential) has been reported. ${ }^{4}$ The pathogenesis of OIP remains a mystery,

\footnotetext{
Address for Correspondence: Yiannis Petros Dimopoulos, Department of Pathology and Laboratory Medicine, MedStar Georgetown University Hospital, USA e-mail: Yiannispetros.dimopoulos@medstar.net

Received: January 28, 2021 Accepted: April 23, 2021 • DOI: 10.5152/balkanmedj.2021.21187

Available at www.balkanmedicaljournal.org

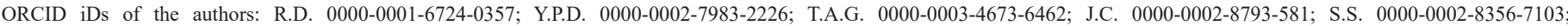
M.O. 0000-0002-1847-5165.

Cite this article as:

Derakhshandeh R, Dimopoulos YP, Goodglick TA, Chanine J, Sabet S, Özdemirli M. Single institutional experience on orbital inflammatory pseudotumor: Diagnostic and management challenge. Balkan Med J. 2021;38(4):239-243.

Copyright@Author(s)-Available online at http://balkanmedicaljournal.org/
} 
but infectious and immune-mediated etiologies have been suggested. ${ }^{5}$ One proposed theory is molecular mimicry, where a foreign microbial antigen has structural similarities with self-antigens-leading to immune system activation and response. In the case of auto-immunity, continuous exposure to self-antigen may lead to chronic inflammation. ${ }^{6}$ OIP has been observed in association with a variety of other immune diseases, and an immunemediated pathophysiology is also strongly suggested with the observations of increased cytokines associated with the disease and the rapid response to immunosuppressive agents. ${ }^{7}$ The current concept of OIP defines it as an idiopathic tumor-like inflammation arising from a polymorphic inflammatory cellular response and a fibrovascular tissue reaction. Histopathologic analysis typically shows chronic inflammation and fibrosis. ${ }^{8}$

Patients most commonly present in their third to fifth decades with relatively sudden onset exophthalmos. Moderate to severe orbital pain is reported by at least one-third of the patients, along with lid and conjunctival edema. ${ }^{9}$ OIP is relatively less common in pediatric populations. ${ }^{10}$ Radiologically, OIP lesions present as ill-defined infiltrative lesions involving the orbit. Thus, biopsy and tissue culture are imperative for definite diagnosis. Evaluation and management of patients with OIP is challenging, and the importance of its inclusion in the differential diagnosis of orbital disorders cannot be overstressed. ${ }^{8,10}$

A crucial differential diagnosis that must be excluded is lymphoma. ${ }^{11}$ Clinical presentation somewhat differs between the 2 disorders. Patients with malignant lymphoid tumors present with palpable mass lesions more frequently compared to OIP, which frequently present with swollen eyelid, conjunctival congestion, pain, retinal folds, or hemorrhage. ${ }^{8}$ However, almost one-third of the patients with OIP may morphologically have features of lymphoid neoplasia and require immunophenotypic and molecular studies to exclude lymphoid malignancies. ${ }^{8}$ Additionally, the finding of clonality of B or T-cell receptors in cases pathologically diagnosed as OIP has unknown clinicopathologic significance with regard to recurrence or progression to overt lymphoid neoplasia.

Occasionally, OIP shows an increase in infiltration of IgG4 plasma cells. Some cases which were previously diagnosed as OIP, have been found to be associated with or have features of IgG4-related disease (IgG4-RD). ${ }^{12}$ Increased awareness of IgG4-RD has led to re-classification of these cases of OIP as IgG4-associated orbital inflammation, with important clinical and therapeutic consequences for patients. ${ }^{13}$

In this study, we sought to investigate potential B and T-cell clonality and investigate the prevalence of features suggestive of IgG4$\mathrm{RD}$ in cases pathologically diagnosed as OIP.

\section{MATERIAL AND METHODS}

This study was reviewed and received approval by the Georgetown-MedStar IRB system.
Our pathology department's records over the past 10 years were interrogated for cases with a final diagnosis of OIP. Cases of IP of other organs were excluded.

Pathology reports, hematoxylin and eosin (H\&E) stained slides, immunohistochemically stained slides (CD138 (B-A38, Cell Marque), IgG4 (HP6025, AbD Serotec), IgG (Polyclonal, Dako-Agilent), CD20 (L26, Dako-Agilent), CD3 (Polyclonal, Dako-Agilent) and ALK (ALK-1, Dako-Agilent)) using the Autostainer Link 48 (Dako-Agilent Technologies Santa Clara, $\mathrm{CA}$ ), and polymerase chain reactions (Invivoscribe BioMed primers kits-Invivoscribe, Inc. San Diego, CA along with ABI 3500 Hitachi XL sequencer-Thermo Fisher Scientific) for the investigation of clonality of B (Immune globulin heavy chain/ IgH and Immune globulin kappa chain/Igא) and T (TCR $\beta$ and $\mathrm{TCR} \gamma$ ) cell receptors were reviewed or performed for each case to confirm the diagnosis and investigate the prevalence of concomitant disease.

Additionally, clinical records were evaluated for basic demographics (age, sex) as well as follow-up, with emphasis on disease recurrence or potential progression.

\section{RESULTS}

A total of 13 cases of OIP were identified, with the majority of the cases representing initial presentations and 1 case a disease recurrence. The majority of patients were female (70\%). Age ranged from 23 to 79, with an average age of 51 years. Demographic data of the patients are presented in Table 1.

Pathological findings on H\&E-stained sections consistent with a diagnosis of IP were considered a mixed lymphoplasmacytic infiltrate on a sclerotic background (Figure 1). On morphological basis, none of the cases had overt features of a clonal lymphocytic disorder/lymphoma. Immunohistochemical analysis with CD138, IgG, and IgG4 showed varying numbers of plasma cells in each case. Five cases $(5 / 13,38 \%)$ exhibited relative increase

TABLE 1. Basic Demographic Data of the Patients As Well As the Prevalence of IgG4rD and B and T-Cell Clonality

\begin{tabular}{lc}
\hline Characteristics & $n(\%)$ \\
\hline Total patients & 13 \\
$\quad$ Males & $4(30)$ \\
Females & $9(70)$ \\
Age (in years) & 51 \\
Mean & 55 \\
Median & $23-79$ \\
Range & \\
IgG4 & $5(38)$ \\
IgG4 increased & $2(15)$ \\
IgG4/IgG $>40 \%$ & \\
Clonality & $2(15)$ \\
B cell (IgH, Igא) & $0(0)$ \\
T cell (TCR $\beta$, TCR $\gamma)$ & \\
\hline
\end{tabular}



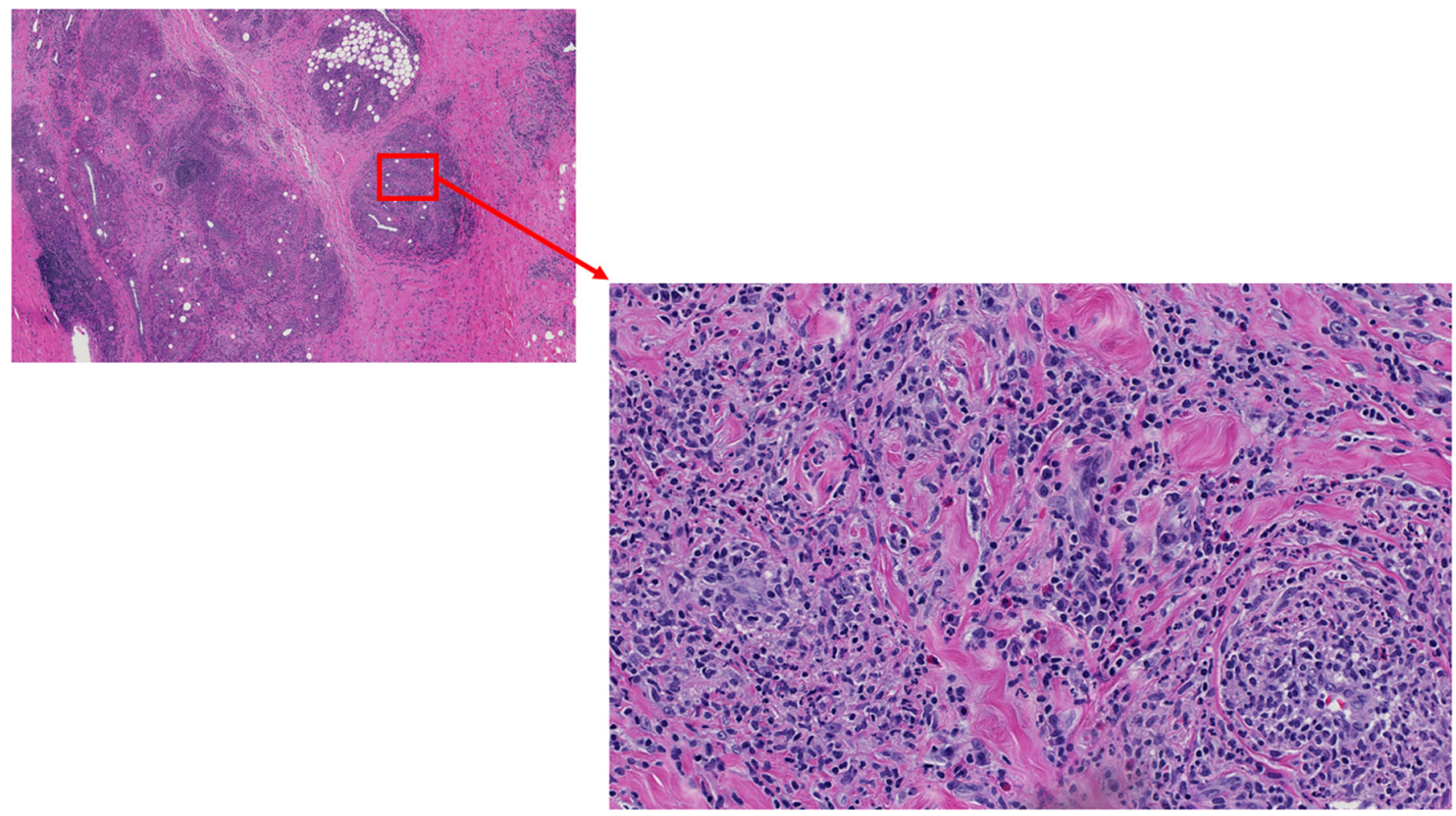

FIG. 1. Typical histopathology of OIP, showing a lymphoplasmacytic and histiocytic infiltrate on a sclerotic background. (Panoramic view and closer view with H\&E stain).

in the presence of IgG4 plasma cells, with 2 cases reaching a ratio of $\mathrm{IgG} 4 / \mathrm{IgG}>40 \%$, suggestive of a diagnosis of IgG4-RD involving the orbit $(2 / 13,15 \%)$ (Figure 2$)$. However, no cases had overt features of IgG4-RD, such as phlebitis or storiform fibrosis. ${ }^{14}$

None of the cases had histologic features of IMT or showed nuclear reactivity for ALK on immunohistochemistry.

PCR performed on formalin fixed-paraffin embedded tissue from each case was used to identify potential clonality of the lymphocytes present. This analysis showed clonal B cell (IgH and Igא) populations in 2 cases $(2 / 13,15 \%)$. The cases that exhibited clonality based on PCR analysis were separate from the cases that showed an increase in IgG4 plasma cells. Additionally, PCR analysis in the patient with disease recurrence failed to reveal clonality (Table 1).

None of the patients showed signs of overt lymphoma pre-operatively (i.e., lymphadenopathy, B-symptoms, etc.) or showed signs of developing lymphoid neoplasia or IgG4-RD following surgical procedure (average follow-up time available was 300 days).
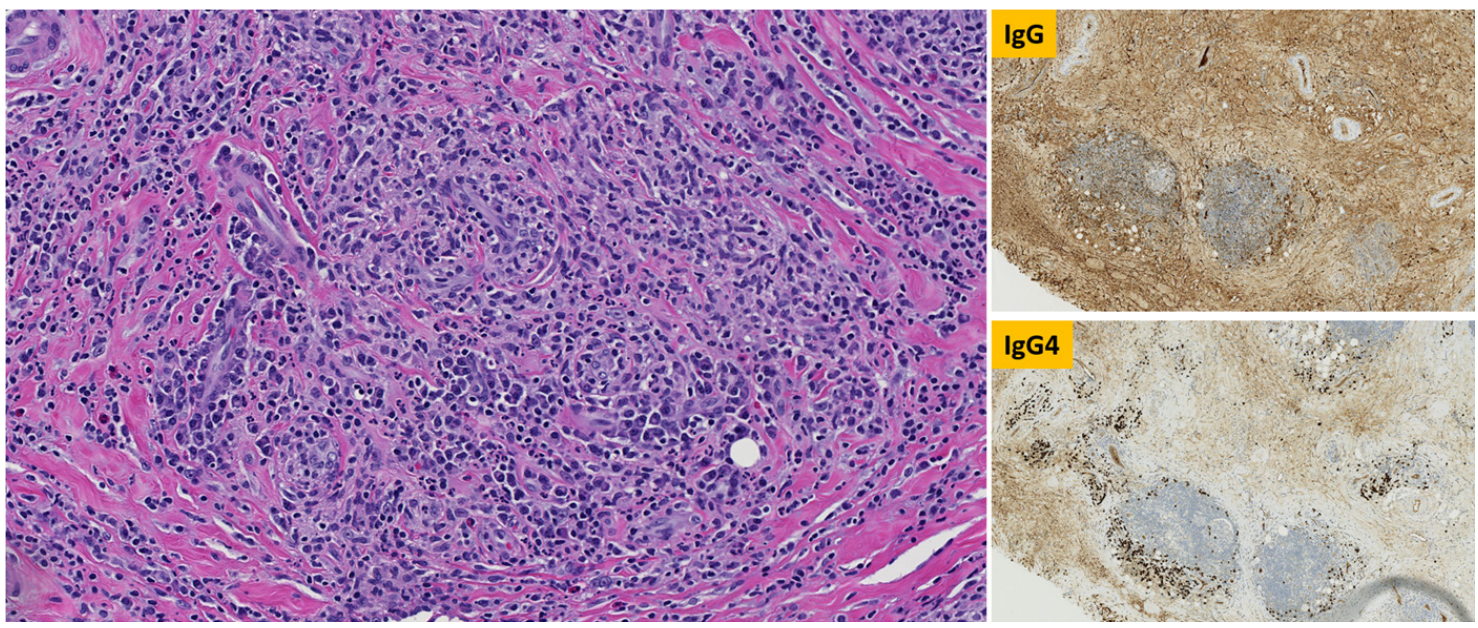

FIG. 2. Example of a case associated with increased IgG4 plasma cells. Numerous plasma cells are seen on an H\&E stain (left). Corresponding lgG and lgG4 immunostains, showing an increase in the IgG4 subtype of plasma cells (right). 
Regarding other concomitant clinical conditions, none of the patients had thyroid disease or thyroid-associated orbitopathy. A total of 5 patients $(5 / 13,38 \%)$ exhibited systemic rheumatologic disease, either known at the time of diagnosis or detected during patient follow-up. These diseases included idiopathic orbital myositis (2 patients), lupus profundus (1 patient), rheumatoid arthritis (1 patient), and granulomatosis with polyangiitis (1 patient).

\section{DISCUSSION}

Despite considerable publications, the basic nature of IP is elusive and it possibly represents a group of lesions with potentially different outcomes. IPs have been described in multiple organs but most commonly reported in the lungs..$^{15} \mathrm{OIP}$, also known as an idiopathic orbital inflammatory syndrome, is a commonly occurring IP in the head and neck region. ${ }^{16}$ Previous studies have shown that orbital tissues from patients with OIP contain high concentrations of retinoids which ultimately lead to activation and release of cytokines. Such cytokine expression makes the orbit prone to respond to any additional systemic or local pro-inflammatory signals, further supporting the potential inflammatory nature of this condition. ${ }^{17}$

Since clinical and radiological manifestations could mimic a neoplastic process, histological evaluation is crucial to exclude other conditions. Proper recognition of this entity is clinically important for the determination of prognosis and formulation of an appropriate treatment plan. Additionally, given the known association with the development of systemic rheumatologic conditions following a diagnosis of OIP, ${ }^{18,19}$ patients should be followed up carefully for other autoimmune conditions.

Regardless of location, all IPs share certain histologic features. Histologically, OIP is characterized by a lymphoplasmacytic and histiocytic infiltrate on variably fibrotic stroma. ${ }^{16}$ No evidence of clonal $\mathrm{T}$ or B-cell receptor has been reported before.

Depending on the site of origin, IPs represent a heterogenous group of lesions of differing nature and outcomes. For example, Cheuk et al. postulated that some IPs arising in the liver or spleen are EBV-associated follicular dendritic cell neoplasms and suggested that the term "IP-like follicular dendritic cell tumor" might be a more apt description for this group. ${ }^{20}$

Certain entities enter the differential diagnosis of IP of the orbit. Similar to OIP, IgG4-RD is a fibro-inflammatory condition characterized by tumefactive lesions in multiple body sites. It has a characteristic histopathological appearance, consisting of dense lymphoplasmacytic infiltrate, storiform fibrosis, and obliterative phlebitis, with patients often showing elevated serum IgG4 levels. ${ }^{14}$ IgG4-related ophthalmic disease can involve all orbital structures and has been recognized as a distinct entity since $2004 .{ }^{21}$ Histologically, IgG4-RD is characterized by lymphocyte and plasma cell infiltration with a ratio of IgG4 + cells to $\mathrm{IgG}+$ cells of $40 \%$ or above ${ }^{14}$ Fibrosis and infiltration of lymphocytes and plasma cells are the overlapping features between this entity and OIP. Some cases which were previously diagnosed as OIP have been found to be associated with or have features of
IgG4-RD. ${ }^{12}$ Additionally, a high prevalence of serum IgG4 has been reported in sino-nasal and ventral skull base IP. ${ }^{22}$ A significant proportion (38\%) of patients with OIP whom we examined showed features suggestive but not diagnostic of IgG4-RD. Patients with OIP showing features of IgG4-RD should be evaluated further (serum IgG4, further imaging), to exclude systemic IgG4-RD.

As mentioned previously, IMT is in the spectrum of IP, with similar histologic and imaging findings, and thus enters the differential diagnosis. IMT has been considered a neoplastic counterpart of IP. The lesion is composed of neoplastic proliferation of myofibroblastic cells accompanied by lymphocytes, plasma cells, and eosinophils. Coffin et al. showed that half of IMTs aberrant cytoplasmic ALK expression in soft tissue and most orbital IMTs have been negative for ALK immunostaining. ${ }^{23}$ Furthermore, nuclear expression of p53 was described in $80 \%$ of IMTs. As a result, ALK and 553 expression may predict progression to IMT. ${ }^{24}$ None of the cases in our cohort exhibited ALK reactivity.

OIP may show histopathologic overlap with lymphomas arising in the orbit. Accurate differentiation between these 2 entities is clinically essential before treatment. Molecular studies could be helpful in differentiating between these 2 entities, as OIP mostly shows a polyclonal population of $\mathrm{T}$ and $\mathrm{B}$ cells in contrast to lymphoma, in which a clonal population of $\mathrm{T}$ or $\mathrm{B}$ cells is present. Matsuo et al. reported a patient who developed systemic lymphoma 17 years after being diagnosed with bilateral OIP. They suggested that the patient might have been predisposed genetically or immunologically to develop bilateral OIP and systemic lymphoma. ${ }^{25}$ A small but significant proportion (15\%) of our cases showed evidence of clonal B-cell receptor rearrangement (IgH and Igא). Although demonstration of clonality in OIP currently has no known long-term implication, this finding should prompt close follow-up of the patient to detect potential future development of lymphoma.

Thirteen cases showing characteristic histopathological features of OIP in our institution were reviewed to better understand the pathogenesis underlying this disease and investigate the frequency of concomitant pathology. A small but significant percentage showed B-cell clonality on PCR analysis of B-cell receptors. Other cases had histologic features suggestive of IgG4-RD. None of the cases showed ALK expression by immunohistochemistry. Clinically, none of the patients had or developed overt features of lymphoid neoplasia or IgG4-RD. None of the patients had thyroid disease. However, a significant percentage was found in association with systemic rheumatologic conditions.

In conclusion, our data emphasizes that OIP should be a diagnosis of exclusion, with careful exclusion of systemic rheumatologic conditions. Findings of clonality of B or T-cell receptors, as well as features of IgG4-RD should prompt close clinical follow-up to detect potential development of systemic lymphoma or IgG4-RD. Given the relative obscure pathogenesis of the disease, additional studies are warranted to promote clinician awareness and gain further knowledge of concomitant diseases. 
Ethics Committee Approval: This study was reviewed and approved by the MedStar/ Georgetown IRB (STUDY00002444, September 9, 2020). No personal identifiable or Protected Health Information was released in the manuscript.

Patient Consent for Publication: N/A.

Data-sharing Statement: The authors confirm that all data supporting the findings of this study are available within the article.

Author Contributions: Concept - R.D., Y.P.D., M.Ö.; Design - R.D., Y.P.D., M.Ö.; Supervision - M.Ö.; Materials - R.D., Y.P.D.; Data Collection and/or Processing - R.D., Y.P.D., J.C.; Analysis and/or Interpretation - R.D., Y.P.D., S.S., T.A.G., J.C., M.Ö.; Literature Review - R.D., Y.P.D., S.S., T.A.G., J.C., M.Ö.; Writing - R.D., Y.P.D.; Critical Review - S.S., T.A.G., J.C., M.Ö.

Acknowledgments: The authors would like to thank Dr. Norio Azumi, MD, PhD for his support of this study as chair of the pathology department.

Conflict of Interest: The authors have no conflicts of interest to declare.

Funding: The authors declared that this study had received no financial support.

\section{REFERENCES}

1. Surabhi VR, Chua S, Patel RP, et al. Inflammatory myofibroblastic tumors: current update. Radiol Clin North Am. 2016;54:553-563. [CrossRef]

2. Umiker WO, Iverson L. Postinflammatory tumors of the lung; report of four cases simulating xanthoma, fibroma, or plasma cell tumor. J Thorac Surg. 1954;28:55-63. [CrossRef]30758-3)

3. Kamili MA, G A, Dar IH, et al. Orbital pseudotumor. Oman J Ophthalmol. 2009;2:9699. [CrossRef]

4. Mahr MA, Salomao DR, Garrity JA. Inflammatory orbital pseudotumor with extension beyond the orbit. Am J Ophthalmol. 2004;138:396-400. [CrossRef]

5. Chaudhry IA, Shamsi FA, Arat YO, Riley FC. Orbital pseudotumor: distinct diagnostic features and management. Middle East Afr J Ophthalmol. 2008;15:17-27. [CrossRef]

6. Das D, Deka P, Bhattacharjee K, et al. Idiopathic inflammatory diseases of orbit and ocular adnexa: histopathological and immunochemical analysis. Indian J Ophthalmol. 2019;67:1993-1995. [CrossRef]

7. Gordon LK. Orbital inflammatory disease: a diagnostic and therapeutic challenge. Eye (Lond). 2006;20:1196-1206. [CrossRef]

8. Yan J, Wu Z, Li Y. The differentiation of idiopathic inflammatory pseudotumor from lymphoid tumors of orbit: analysis of 319 cases. Orbit. 2004;23:245-254. [CrossRef]

9. Yeşiltaş YS, Gündüz AK. Idiopathic orbital inflammation: review of literature and new advances. Middle East Afr J Ophthalmol. 2018;25:71-80. [CrossRef]
10. Spindle J, Tang SX, Davies B, et al. Pediatric idiopathic orbital inflammation: clinical features of 30 cases. Ophthal Plast Reconstr Surg. 2016;32:270-274. [CrossRef]

11. Ren J, Yuan Y, Wu Y, Tao X. Differentiation of orbital lymphoma and idiopathic orbital inflammatory pseudotumor: combined diagnostic value of conventional MRI and histogram analysis of ADC maps. BMC Med Imaging. 2018;18:6. [CrossRef]

12. Chougule A, Bal A. IgG4-related inflammatory pseudotumor: A systematic review of histopathological features of reported cases. Mod Rheumatol. 2017;27:320-325. [CrossRef]

13. Lee CS, Harocopos GJ, Kraus CL, et al. IgG4-associated orbital and ocular inflammation. J Ophthal Inflamm Infect. 2015;5:15. [CrossRef]

14. Obiorah IE, Henao Velasquez A, Özdemirli M. The clinicopathologic spectrum of IgG4-related disease. Balk Med J. 2018;35:292-300. [CrossRef]

15. Kaitoukov Y, Rakovich G, Trahan S, Grégoire J. Inflammatory pseudotumour of the lung. Can Respir J. 2011;18:315-317. [CrossRef]

16. Tao J, Zhou ML, Zhou SH. Inflammatory myofibroblastic tumors of the head and nec. Int J Clin Exp Med. 2015;8:1604-1610.

17. Kahana A. Orbital inflammatory disorders: new knowledge, future challenges. Curr Opin Ophthalmol. 2021;32:255-261. [CrossRef]

18. Rico M, Díaz-López JB, Peña J, Oliva-Nacarino P. Latent orbital pseudotumor secondary to systemic lupus erythematosus. Clin Case Rep. 2016;4:1065-1067. [CrossRef]

19. Tsukikawa M, Lally SE, Shields CL, et al. Idiopathic orbital pseudotumor preceding systemic inflammatory disease in children. J Pediatr Ophthalmol Strabismus. 2019;56:373-377. [CrossRef]

20. Zhang BX, Chen ZH, Liu Y, Zeng YJ, Li YC. Inflammatory pseudotumor-like follicular dendritic cell sarcoma: A brief report of two cases. World J Gastrointest Oncol. 2019;11:1231-1239. [CrossRef]

21. Mejico LJ. IgG4-related ophthalmic disease. Saudi J Ophthalmol. 2015;29:53-56. [CrossRef]

22. Ryu G, Cho HJ, Lee KE, et al. Clinical significance of IgG4 in sinonasal and skull base inflammatory pseudotumor. Eur Arch Otorhinolaryngol. 2019;276:2465-2473. [CrossRef]

23. Coffin CM, Hornick JL, Fletcher CD. Inflammatory myofibroblastic tumor: comparison of clinicopathologic, histologic, and immunohistochemical features including ALK expression in atypical and aggressive cases. Am J Surg Pathol. 2007;31:509520. [CrossRef]

24. Yamamoto H, Oda Y, Saito T, et al. p53 mutation and MDM2 amplification in inflammatory myofibroblastic tumours. Histopathology. 2003;42:431-439. [CrossRef]

25. Matsuo T, Sato Y, Kuroda R, Matsuo N, Yoshino T. Systemic malignant lymphoma 17 years after bilateral orbital pseudotumor. Jpn J Ophthalmol. 2004;48:503-506. [CrossRef] 